\title{
Sex- and age-specific trends in antibiotic resistance patterns of Escherichia coli urinary isolates from outpatients
}

\author{
Jessina C McGregor ${ }^{1 *}$, Miriam R Elman ${ }^{1}$, David T Bearden ${ }^{1}$ and David H Smith²
}

\begin{abstract}
Background: Urinary tract infections (UTIs) are one of the most common infections treated in ambulatory care settings, however the epidemiology differs by age and sex. The incidence of UTI is far greater in females than males, and infection in pediatric patients is more often due to anatomical abnormalities. The purpose of this research was to describe age- and sex-specific trends in antibiotic susceptibility to common urinary anti-infectives among urinary isolates of Escherichia coli from ambulatory primary care patients in a regional health maintenance organization.
\end{abstract}

Methods: Clinical microbiology data were collected for all urine cultures from patients with visits to primary care clinics in a regional health maintenance organization between 2005 and 2010. The first positive culture for E. coli tested for antibiotic susceptibilities per patient per year was included in the analysis dataset. The frequency of susceptibility to ampicillin, amoxicillin-clavulanate, ciprofloxacin, nitrofurantoin, and trimethoprim/sulfamethoxazole (TMP/SMX) was calculated for male and female patients. The Cochrane-Mantel-Haenzel test was used to test for differences in age-stratified susceptibility to each antibiotic between males and females.

Results: A total of 43,493 E. coli isolates from 34,539 unique patients were identified for study inclusion. After stratifying by age, E. coli susceptibility to ampicillin, amoxicillin-clavulanate, ciprofloxacin, and nitrofurantoin differed significantly between males and females. However, the magnitude of the differences was less than $10 \%$ for all strata except amoxicillin-clavulanate susceptibility in E. coli isolated from males age 18-64 compared to females of the same age.

Conclusions: We did not observe clinically meaningful differences in antibiotic susceptibility to common urinary anti-infectives among $E$. coli isolated from males versus females. These data suggest that male sex alone should not be used as an indication for empiric use of second-line broad-spectrum antibiotic agents for the treatment of UTIs.

Keywords: Urinary tract infection, Urinary anti-infectives, Escherichia coli

\section{Introduction}

Urinary tract infections (UTIs) are one of the most commonly treated bacterial infections and account for over 10 million ambulatory care visits annually in the United States [1]. Antibiotic treatment is typically selected empirically, based on the patient clinical presentation, medical history and local patterns of antibiotic susceptibility [2]. Because the incidence of UTIs is significantly greater

\footnotetext{
*Correspondence: mcgregoj@ohsu.edu

'Department of Pharmacy Practice, College of Pharmacy, Oregon State University/Oregon Health \& Science University, 3303 SW Bond Ave., CH12C, Portland, OR 97239, USA

Full list of author information is available at the end of the article
}

among women, much of the research has focused on women. Thus there exists a paucity of research on UTIs in men. As such, guidelines on the diagnosis, treatment, and management of UTIs focus largely on infection in women [2-5].

The incidence, presentation, and course of infection for UTIs in men and women differ in large part due to anatomical differences. For the treatment of acute uncomplicated cystitis in women, trimethoprim/sulfamethoxazole (TMP/SMX), nitrofurantoin, fosfomycin, and pivmecillinam are recommended first-line empiric therapies $[2,6]$. In men, because prostate involvement occurs

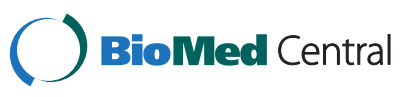


in roughly $90 \%$ of cases, an empiric agent should be selected that achieves therapeutic concentrations in prostatic tissues (e.g., trimethoprim or ciprofloxacin) $[7,8]$.

While historically it was believed that the causative organism in UTIs differed between men and women, more recent data has shown that for both sexes the primary causative pathogen is Escherichia coli, which accounts for $75-90 \%$ of UTIs $[8,9]$. With the increases in antibiotic resistance among E. coli and other Enterobacteriaceae over the past several decades, surveillance data have become critical for appropriate empiric selection of antibiotic therapy. U.S. guidelines specify that TMP/SMX should be avoided for empiric treatment of uncomplicated acute cystitis or pyelonephritis in populations where non-susceptibility to this agent exceeds $20 \%$ in uropathogens [2].

While some surveillance studies have identified significant differences in the frequency of susceptibility to common urinary anti-infectives between isolates collected from male and female patients, this has not been consistently observed $[10,11]$. The objective of this study was to describe age- and sex-specific antibiotic susceptibility patterns for common urinary anti-infectives among E. coli urine isolates using six years of data from a large ambulatory primary care patient population.

\section{Methods}

\section{Study design and patient population}

We conducted a cross-sectional study of urinary E. coli isolates from outpatients of Kaiser Permanente Northwest (KPNW) primary care clinics. KPNW is a regional health maintenance organization that serves over 485,000 members in northwest Oregon and southwest Washington. Primary care clinics were defined as non-specialty care clinics within the Family Practice, Internal Medicine, or Pediatrics departments; all primary care clinics were included. Urine cultures positive for E. coli that were drawn from patients with visits in the primary care clinics between January 1, 2005 and December 31, 2010 were eligible for inclusion in the analysis. Cultures in which less than 10,000 colonies/mL were identified or 3 or more organisms were isolated were excluded. The analysis dataset was then limited to the first isolate tested for antibiotic susceptibilities per patient and year to minimize potential bias resulting from repeat culturing [12].

\section{Data collection}

Data were electronically extracted from the virtual data warehouse maintained by the Kaiser Permanente Center for Health Research. Collected data included patient demographics, department in which the clinic visit occurred, and all clinical microbiology data for urine cultures. Approval for this study was obtained from the KPNW institutional review board.

\section{Data analysis}

The frequency of susceptibility to ampicillin, amoxicillinclavulanate, ciprofloxacin, nitrofurantoin, and trimethoprim/sulfamethoxazole (TMP/SMX) was calculated for $E$. coli isolates stratified by patient sex and year. The Cochrane-Armitage test for trend was used to identify changes in the frequency of susceptibility to each antibiotic over time independently among males and females. Patient age at the time of culture was categorized as less than 18 years, 18-64 years, and 65 years and older. The frequency of susceptibility to each antibiotic was also compared across age categories among males and females using the Cochran-Mantel-Haenszel test. An alpha level less than or equal to 0.05 was the statistical significance level for all analyses and data were analyzed with SAS (version 9.2, SAS Corporation). Clinically significant differences were defined as differences of $10 \%$ or greater.

\section{Results}

\section{Description of study sample}

During the study time frame 190,396 urine cultures were performed at primary care clinic visits; 70,180 were positive for $E$. coli and 57,550 of those were tested for antibiotic susceptibilities. After restricting the data to the first isolate per patient per year, 43,493 isolates from 34,539 unique patients remained in the final analysis data set. Table 1 describes the demographics of these patients. Of the included isolates, 2,520 (5.8\%) were from male patients.

\section{Antibiotic susceptibility of urinary Escherichia coli isolates by patient sex}

Overall, $66.0 \%(1,664 / 2,520)$ of $E$. coli isolated from males and $66.3 \%(27,175 / 40,971)$ from females were

Table 1 Patient characteristics based on first $E$. coli isolate from urine specimen in KPNW outpatients 2005-2010

\begin{tabular}{lll}
\hline Characteristic & \multicolumn{2}{c}{$\mathbf{n = 3 4 5 3 9}$} \\
\hline Mean age (SD) & 32265 & \\
Female & & \\
Ethnicity & 1067 & $(3.1)$ \\
$\quad$ Hispanic & 16984 & $(49.2)$ \\
Non-Hispanic & 16488 & $(47.7)$ \\
Unknown/missing & & \\
Race & 20974 \\
White & 377 & $(60.7)$ \\
Black & 108 & $(1.1)$ \\
Native American/Alaskan Native & 1411 & $(0.3)$ \\
Asian & 46 & $(4.1)$ \\
Pacific Islander/Hawaiian & 767 & $(0.1)$ \\
Other & 10856 & $(2.2)$ \\
Unknown & & $(31.4)$ \\
\hline
\end{tabular}

${ }^{a}$ Data are No. (\%) unless otherwise indicated. 
susceptible to ampicillin. Amoxicillin-clavulanate susceptibility was 56.9\% (484/850) among males and 67.3\% (9,291/ 13,798) among females; however, it should be noted that testing for susceptibility to amoxicillin-clavulanate was routinely performed and reported only for ampicillin nonsusceptible $E$. coli. Ciprofloxacin susceptibility for the E. coli isolates was $93.2 \%(2,292 / 2,458)$ among males and $95.9 \%(37,900 / 39,524)$ among females, and nitrofurantoin susceptibility was $96.4 \%(2,430 / 2,520)$ among males and 97.6\% $(39,979 / 40,968)$ among females. For TMP/SMX, 86.3\% $(2,176 / 2,520)$ of $E$. coli isolated from males were susceptible compared to $84.7 \%(34,674 /$ 40,958) among females.

Trends in antibiotic susceptibility of urinary Escherichia coli isolates over time

Figure 1 presents the susceptibility of $E$. coli to each antibiotic by sex and year. Susceptibility to amoxicillinclavulanate and TMP/SMX decreased significantly over time among both males and females. Ciprofloxacin susceptibility also decreased significantly over time among females, but not males. Table 2 presents the age stratified antibiotic susceptibilities for E. coli isolated from males and females. The age-specific susceptibilities differed significantly between males and females for all antibiotics except TMP/SMX.

\section{Discussion}

In this study, we observed statistically significant differences between males and females in the age-specific susceptibilities of $E$. coli to ampicillin, amoxicillin-clavulanate, ciprofloxacin, and nitrofurantoin. Urinary E. coli isolates from male patients tended to exhibit increased antibiotic resistance than isolates from female patients. Despite the statistical significance of time trends and differences in age-specific susceptibilities, the magnitude of these differences was generally less than $5 \%$ and thus may not represent clinically meaningful differences. The exception was susceptibility to amoxicillin-clavulanate, where susceptibility was roughly $10 \%$ lower in males age 18 to 64 years than females in the same age group. Yet these differences should be interpreted cautiously because susceptibility to amoxicillin-clavulanate was only provided for ampicillin non-susceptible $E$. coli isolates in the current analysis. If all ampicillin susceptible isolates are assumed to be amoxicillin-clavulanate susceptible, then the magnitude of (a) Ampicillin

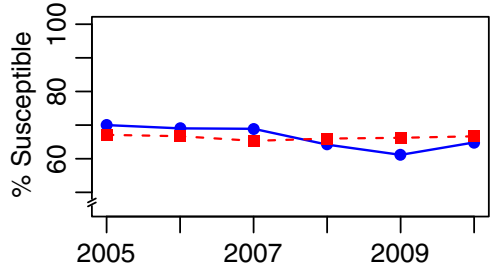

(c) Ciprofloxacin

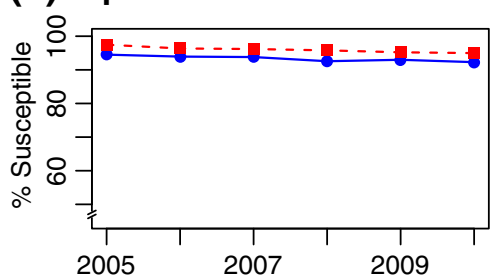

\section{(b) Amoxicillin clavulanate}

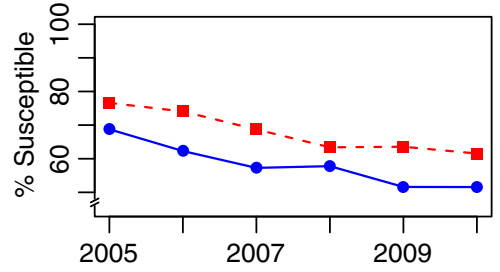

(d) Nitrofurantoin

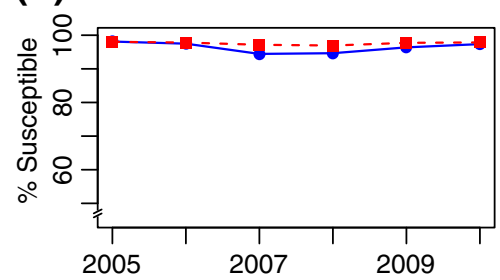

(e) Trimethoprim/sulfamethoxazole
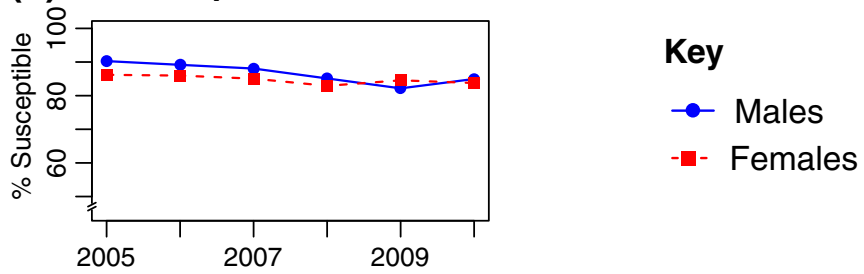

Figure 1 Susceptibility to urinary anti-Infectives by sex and year for $E$. coli cultured from urine. For all figures, males shown as blue solid line, females as red dashed line. $\mathbf{a}$, ampicillin susceptibility (test for trend: males, $\mathrm{p}=0.058$; females, $\mathrm{p}=0.6256$ ); $\mathbf{b}$, amoxicillin clavulanate susceptibility (test for trend: males, $p=0.0006$; females, $p<0.0001$ ); $\mathbf{c}$, ciprofloxacin susceptibility (test for trend: males, $p=0.1445$; females, $p<0.0001$ ); d, nitrofurantoin susceptibility (test for trend: males, $p=0.4967$; females, $p=0.5508$ ); $\mathbf{e}$, trimethoprim/sulfamethoxazole susceptibility (test for trend: males, $p=0.0004$; females, $p<0.0001$ ). 
Table 2 Susceptibility to urinary anti-infectives by sex and age for $E$. coli cultured from urine*

\begin{tabular}{|c|c|c|c|c|}
\hline & \multicolumn{3}{|c|}{ Age (years) } & \multirow{2}{*}{$\begin{array}{c}\mathrm{CMH} \\
\text { p-value }\end{array}$} \\
\hline & $<18$ & $18-64$ & $>64$ & \\
\hline Ampicillin & & & & $<0.0001$ \\
\hline Male & $104(58.8)$ & $852(68.5)$ & $708(64.4)$ & \\
\hline Female & $2564(62.6)$ & $16988(65.4)$ & $7623(69.9)$ & \\
\hline Amoxicillin clavulanate & & & & $<0.0001$ \\
\hline Male & $45(64.3)$ & $214(55.6)$ & $225(57.0)$ & \\
\hline Female & $1113(73.1)$ & $6040(67.5)$ & $2138(64.2)$ & \\
\hline Ciprofloxacin & & & & $<0.0001$ \\
\hline Male & $112(97.4)$ & $1201(96.5)$ & $979(89.1)$ & \\
\hline Female & $2613(98.4)$ & $25161(96.9)$ & $10126(92.8)$ & \\
\hline Nitrofurantoin & & & & $<0.0001$ \\
\hline Male & $174(98.3)$ & $1214(97.6)$ & $1042(94.8)$ & \\
\hline Female & $4023(98.2)$ & $25413(97.9)$ & $10543(96.6)$ & \\
\hline Trimethoprim/Sulfamethoxazole & & & & 0.3888 \\
\hline Male & $147(83.1)$ & 1090 (87.6) & $939(85.4)$ & \\
\hline Female & $3479(84.9)$ & $21909(84.4)$ & $9286(85.1)$ & \\
\hline
\end{tabular}

*Represents the first isolate for each patient and year.

CMH: Cochrane Maentel Haenszel correlation test.

the differences between males and females would be less than $5 \%$ in all age categories (difference not statistically significant).

While few other studies have explored the differences in antibiotic susceptibility of uropathogens isolated from male and female ambulatory patients, our findings are consistent with the trends observed in the literature. A recent 10-year study of community UTI in Portuguese patients also identified differences in antibiotic susceptibility by patient sex. The authors reported that urinary isolates of $E$. coli were significantly more resistant to fluoroquinolones, penicillins, nitrofurantoin, and first and second generation cephalosporins among men compared to women [13]. Another study focused on pediatric patients, also identified significantly higher resistance to TMP/SMX and ciprofloxacin in male versus female patients [14]. The NAUTICA surveillance study of outpatient UTIs reported greater antibiotic resistance to ciprofloxacin, levofloxacin, and TMP/SMX among all urinary isolates from U.S. and Canadian male patients [15]. In the CANWARD study, antibiotic susceptibility among all E. coli isolates (not limited to urine isolates) collected from Canadian tertiary medical centers were compared and resistance was also observed to be significantly higher to ciprofloxacin, levofloxacin, and TMP/SMX in isolates collected from male patients versus female patients [11].

The prevalence and susceptibilities of antibioticresistant bacteria varies widely by geographic region, thus these data may not be generalizable to all regions. Local surveillance data of antibiotic susceptibilities would be needed to validate these findings in different patient populations. It should be noted that in our study resistance to TMP/SMX did not exceed $20 \%$, as it does in many other regions of the U.S., thus in this patient setting TMP/SMX may be a viable empiric treatment option in both males and female patients. Additionally, antibiotic susceptibilities may differ by other patient or infection characteristics (e.g., type of UTI). These data were not available for analysis in this study and further investigation in this area is needed. Also, because clinical signs and symptoms of infection were not available and because not all suspected infections are cultured, the use of all urinary microbiology from this population cultures may not represent all true infections.

Currently there exists insufficient data to inform the development of evidence-based guidelines for the treatment of community UTIs in men. UTIs in adult males are considered complicated infections according to most clinical definitions [16-18]. Treatment recommendations vary from longer durations of therapy to the selection of broad-spectrum agents such as fluoroquinolones [6]. These recommendations are driven by the high proportion of UTIs in men with prostate involvement and concerns surrounding antibiotic resistance to TMP/SMX $[7,8]$. Previous research among Swiss outpatients has demonstrated the selection of fluoroquinolones versus TMP/SMX for the treatment of UTI is often influenced by nonclinical factors [19]. In this study, TMP/SMX, a recommended first-line agent for UTIs in both children and adults, the differences in susceptibility between E. coli isolated from males and females were neither 
statistically nor clinically significant and resistance rates are below 20\%. Consequently, in this population, there is no evidence that male sex alone should be an indication for empiric selection of a second-line broadspectrum antibiotic agent for the treatment of UTI. While more research is needed on treatment effectiveness of different regimens used to treat community UTIs in men, population-specific antibiotic susceptibility data are a necessary component in the empiric antibiotic selection process.

\section{Conclusions}

In the era of increasing antibiotic resistance, prudent use of antibiotics is critical to prolong the clinical effectiveness of existing agents. Excessive use of broad-spectrum agents, such as fluoroquinolones, increases the evolutionary selective pressures that drive the increasing prevalence of resistance. These data suggest that firstline urinary anti-infectives such as TMP/SMX may be effective agents for treating UTIs in men. While more data are needed, clinicians should use local surveillance data to guide the prudent, empiric selection of antibiotic therapy for UTIs.

\section{Abbreviations}

UTI: Urinary Tract Infection; KPNW: Kaiser Permanente Northwest;

TMP/SMX: trimethoprim/sulfamethoxazole.

\section{Competing interests}

The authors declare that they have no competing interests.

\section{Authors' contributions}

JCM participated in the conception and design of the study; the acquisition, statistical analysis, and interpretation of the data; and the drafting of the manuscript. JCM, MRE, DTB, and DHS assisted with the interpretation of the data and participated in the critical revision of the manuscript. All authors read and approved the final manuscript.

\section{Author details}

${ }^{1}$ Department of Pharmacy Practice, College of Pharmacy, Oregon State University/Oregon Health \& Science University, 3303 SW Bond Ave., CH12C, Portland, OR 97239, USA. ${ }^{2}$ Kaiser Permanente Northwest Center for Health Research, Portland, OR, USA.

Received: 16 July 2012 Accepted: 20 February 2013

Published: 22 February 2013

\section{References}

1. Schappert $S M$, Rechtsteiner $E A$ : Ambulatory medical care utilization estimates for 2007. Vital Health Stat 2011, 13:1-38.

2. Gupta K, Hooton TM, Naber KG, Wullt B, Colgan R, Miller LG, Moran GJ, Nicolle LE, Raz R, Schaeffer AJ, Soper DE: International clinical practice guidelines for the treatment of acute uncomplicated cystitis and pyelonephritis in women: a 2010 update by the infectious diseases society of america and the European society for microbiology and infectious diseases. Clin Infect Dis 2011, 52:e103-120.

3. Gupta K, Sahm DF, Mayfield D, Stamm WE: Antimicrobial resistance among uropathogens that cause community-acquired urinary tract infections in women: a nationwide analysis. Clin Infect Dis 2001, 33:89-94.

4. Karlowsky JA, Kelly LJ, Thornsberry C, Jones ME, Sahm DF: Trends in antimicrobial resistance among urinary tract infection isolates of Escherichia coli from female outpatients in the United States. Antimicrob Agents Chemother 2002, 46:2540-2545.
5. Hooton TM, Johnson C, Winter C, Kuwamura L, Rogers ME, Roberts PL, Stamm WE: Single-dose and three-day regimens of ofloxacin versus trimethoprim-sulfamethoxazole for acute cystitis in women. Antimicrob Agents Chemother 1991, 35:1479-1483.

6. European Association of Urology: Guidelines on Urological Infections. Arnhem, The Netherlands:; 2011. http://www.uroweb.org/gls/pdf/ 15_Urological_Infections.pdf.

7. Ulleryd P, Zackrisson B, Aus G, Bergdahl S, Hugosson J, Sandberg T: Prostatic involvement in men with febrile urinary tract infection as measured by serum prostate-specific antigen and transrectal ultrasonography. BJU Int 1999, 84:470-474.

8. Ulleryd P: Febrile urinary tract infection in men. Int J Antimicrob Agents 2003, 22(Suppl 2):89-93.

9. Gupta K, Hooton TM, Stamm WE: Increasing antimicrobial resistance and the management of uncomplicated community-acquired urinary tract infections. Ann Intern Med 2001, 135:41-50.

10. Zhanel GG, Hisanaga TL, Laing NM, DeCorby MR, Nichol KA, Weshnoweski B, Johnson J, Noreddin A, Low DE, Karlowsky JA, Hoban DJ: Antibiotic resistance in Escherichia coli outpatient urinary isolates: final results from the North American Urinary Tract Infection Collaborative Alliance (NAUTICA). Int J Antimicrob Agents 2006, 27:468-475.

11. Lagace-Wiens PR, Simner PJ, Forward KR, Tailor F, Adam HJ, Decorby M, Karlowsky J, Hoban DJ, Zhanel GG: Analysis of 3789 in- and outpatient Escherichia coli isolates from across Canada-results of the CANWARD 2007-2009 study. Diagn Microbiol Infect Dis 2011, 69:314-319.

12. Hindler JF, Barton M, Callihan DR, Erdman SM, Evangelista AT, Jenkins SG, Johnston J, Master R, McGowan J, John E, Nimmo G, Stelling J: Analysis and Presentation of Cumulative Antimicrobial Susceptibility Test Data; Approved Guideline--Third Edition (M39-A3). In Book Analysis and Presentation of Cumulative Antimicrobial Susceptibility Test Data; Approved Guideline--Third Edition (M39-A3), Volume 26. Wayne, Pennsylvania: Clinical and Laboratory Standards Institute; 2009.

13. Linhares I, Raposo T, Rodrigues A, Almeida A: Frequency and antimicrobial resistance patterns of bacteria implicated in community urinary tract infections: a ten-year surveillance study (2000-2009). BMC Infect Dis 2013, 13:19.

14. Edlin RS, Shapiro DJ, Hersh AL, Copp HL: Antibiotic resistance patterns in outpatient pediatric urinary tract infections. J Urol 2013, .

15. Zhanel GG, Hisanaga TL, Laing NM, DeCorby MR, Nichol KA, Palatnik LP, Johnson J, Noreddin A, Harding GK, Nicolle LE, Hoban DJ: Antibiotic resistance in outpatient urinary isolates: final results from the North American Urinary Tract Infection Collaborative Alliance (NAUTICA). Int J Antimicrob Agents 2005, 26:380-388.

16. Sobel JD, Kaye D: Urinary Tract Infections. In Mandell, Douglas, and Bennett's principles and practice of infectious diseases, Volume 1. 6th edition. Edited by Mandell GL, Douglas RG, Bennett JE, Dolin R. New York: Elsevier/ Churchill Livingstone; 2005:875-905.

17. McCue JD: UTIs in at-risk patients: Are they 'complicated'? Infect Med 1999, 16:533-540.

18. Anderson RU: Treatment of complicated and uncomplicated urinary tract infections. In Antibiotic therapy in urology. Edited by Mulholland SG. Philadelphia: Lippincott-Raven Publishers; 1996:23-37.

19. Stuck AK, Tauber MG, Schabel M, Lehmann T, Suter H, Muhlemann K: Determinants of quinolone versus trimethoprim-sulfamethoxazole use for outpatient urinary tract infection. Antimicrob Agents Chemother 2012, 56:1359-1363.

doi:10.1186/1471-2296-14-25

Cite this article as: McGregor et al:: Sex- and age-specific trends in antibiotic resistance patterns of Escherichia coli urinary isolates from outpatients. BMC Family Practice 2013 14:25. 